\title{
Coalescence of Pendant Droplets on an Inclined Super-hydrophobic Substrate
}

\author{
Basant Singh Sikarwar ${ }^{1}$, Sameer Khandekar ${ }^{2}$, K. Muralidhar ${ }^{3}$ \\ 1: Department of Mechanical Engineering, Indian Institute of Technology Kanpur, India, sikarwar@iitk.ac.in \\ 2: Department of Mechanical Engineering, Indian Institute of Technology Kanpur, India, samkhan@iitk.ac.in \\ 3: Department of Mechanical Engineering, Indian Institute of Technology Kanpur, India, kmurli@iitk.ac.in
}

\begin{abstract}
Dynamics of micro droplets, coalescence underneath an inclined hydrophobic surface is explored, experimentally as well as computationally. Such a situation often arises during dropwise condensation, atmospheric dew formation, condensation in green houses, and metal vapor condensation during enrichment process, etc. Coalescence induced instability in the pendant mode is an effective means of passively enhancing heat transfer coefficient during dropwise condensation. Inclined substrates have natural advantages in terms of rendering effective passive sweeping of drops from the substrate, thereby exposing fresh preferred sites for renucleation. As compared to coalescences of sessile droplets, pendant mode induces flow instabilities at a much faster rate, thereby enhancing the associated heat/ mass transport characteristics. Against this background, the present study reports an experiment involving the coalescence of water drops in pendant mode on an inclined hydrophobic copper substrate of size $20 \mathrm{~mm} \times 20 \mathrm{~mm} \times 2 \mathrm{~mm}$. The substrate has been prepared by developing a monolayer of n-octadecanethiol on it by chemical action. This work focuses on fundamental understanding of the mechanism of drop coalescence underneath an inclined superhydrophobic substrate to provide aid in controlling and promoting dropwise mode of condensation in order to increase efficiency of condenser or to aid in enrichment of heavy liquid metals in closed vacuum condition. The coalescence process gets initiated by the extra available surface energy which gets released in the process. Immediately afterwards, the process of oscillating free surface formed during the coalescence is limited by viscous and inertia forces. The free surface oscillations can last $~ 10$ to 100 milliseconds, depending on the size of droplets and their thermophysical properties. Depending on the local wettability, size of drops which are coalescing, substrate inclination and pinning/de-pinning behavior, droplet instability conditions are generated, which result in its slide-off motion on the substrate.
\end{abstract}

Keywords: Droplet coalescence, pendant mode, hydrophobic surfaces, confocal microscopy, interface shape and dynamics

\section{Introduction}

Drop coalescence is a process by which two or more droplets merge during contact to form a single droplet. It is a common phenomenon in nature and encountered in many fields, ranging from molecular clusters to rain-drop formation in clouds as well as transport process in chemical industry, environment engineering, heat transfer and energy systems (Andrieu et al. 2002; Sikarwar et al. 2012; Bordoloi and Longmire, 2012; Sellier and Trelluyer, 2011).

During laboratory experiments of dropwise condensation process on or underneath hydrophobic surfaces, several researchers (Leach et al. 2006; Rose 2002, Sikarwar et al. 2010) have observed smallest detectable drops that first grow and eventually fall-off, after repeated coalescence with neighboring drops. Much of the research covers the formation of the liquid bridge and the relaxation time of sessile drops during their coalescence. When two droplets touch each other, a liquid bridge between the two drops is formed, due to which a negative curvature or negative pressure is created at point of joining of two liquid drops. This bridge, due to its large curvature, quickly expands under the influence of interfacial stress and the resultant fluid motion pulls two drops together, thereby forming a large drop with smaller net surface area (Kapur and Gaskell, 2007). Hence, the very early time of coalescence commences with a bridge formation between the drops, with a strong vapor-liquid interface curvature, leading in turn, to strong relaxation forces.

Andrieu et al. (2002) studied the experimental description of the kinetics of coalescence of two sessile drops on a horizontal substrate. It was found that the drops slowly 
and exponentially relaxed to an equilibrium hemispherical cap. The characteristic relaxation time was proportional to drop radius at the final equilibrium, and the relaxation time appeared to be nearly $10^{7}$ times larger than the bulk capillary relaxation time. Narhe et al. (2004) used a syringe to position one droplet next to another in an attempt to explore the coalescence event in the sessile mode. Again, exponential relaxation of the composite drop was observed; typical relaxation time decreased with contact angle. The relaxation dynamics was also larger by 5 to 6 orders of magnitude than the bulk hydrodynamic, due to the high dissipation in the contact line vicinity.

From a fundamental transport point of view, droplet coalescence is a fairly complex phenomenon, involving free surfaces, viscous damping and surface tension stabilization. Gravitational body forces may also play a role if the size of the drop is large enough (Narhe et al., 2005). This type of fluid flow involves a topological transition in the flow domain. Coalescence of drops sets in a dynamic process involving characteristic timescales which depend on the geometry and the thermophysical properties of the fluid involved (Blanchette et al., 2009; Wang et al., 2010). Many researchers (Anderie et al 2002; Eggers, 1998; Sikarwar et al. 2012) have reported that the final position of merged drop is approximately at the weighted center of mass resulting from the original two drops before coalescence. Liao et al. (2005) experimentally studied the effect of inclination on coalescence and reported the variation of contact angles before and after coalescence.

Fairly extensive literature is available on the phenomenon of sessile drop coalescence. The dynamics of drop coalescence underneath an inclined hydrophobic surface, i.e. in pendant mode, remains largely unexplored. Such a situation often arises during dropwise condensation, atmospheric dew formation, condensation in green houses, and metal vapor condensation during enrichment process, etc. Droplet coalescence dynamics will eventually lead to enhanced heat transfer during dropwise condensation process (Sikarwar et al. 2010). Coalescence induced instability is an effective means of passively enhancing heat transfer coefficient during dropwise condensation. Inclined substrates have natural advantages in terms of rendering effective passive sweeping of the condensate droplets from the substrate, thereby exposing fresh sites for renucleation. As compared to coalescences of sessile droplets, pendant mode induces flow instabilities at a much faster rate, thereby enhancing the associated heat/ mass transport characteristics. Explicit literature highlighting the relation between pendant mode of droplet coalescence and its effect on heat transfer enhancement is also scarce. Much of the research covers the formation of a liquid bridge and the relaxation time of drop after coalescence in sessile mode.

Against this background, the present study reports an experimental as well as a computational study involving the coalescence of water drops in pendant mode on an inclined hydrophobic copper substrate of size $20 \mathrm{~mm} \times$ $20 \mathrm{~mm} \times 2 \mathrm{~mm}$. The substrate has been prepared by depositing a monolayer of noctadecanethiol on it by chemical action. The process involves thorough cleaning of the substrate, de-greasing and acid etching, preconditioning and desiccating inside a vacuum oven at certain temperature, and finally controlled immersion in a solution of noctadecanethiol in ethanol. In this manner, maximum equilibrium contact angle of $\sim 135^{\circ}$ could be achieved for a water droplet. The process is simple and repeatable and produces good quality super-hydrophobic copper surfaces. Figure 1 shows the photograph of a pendant drop of $25 \mu \mathrm{l}$ water droplet on the prepared surface in horizontal as well as at $15^{\circ}$ inclined surface, respectively.

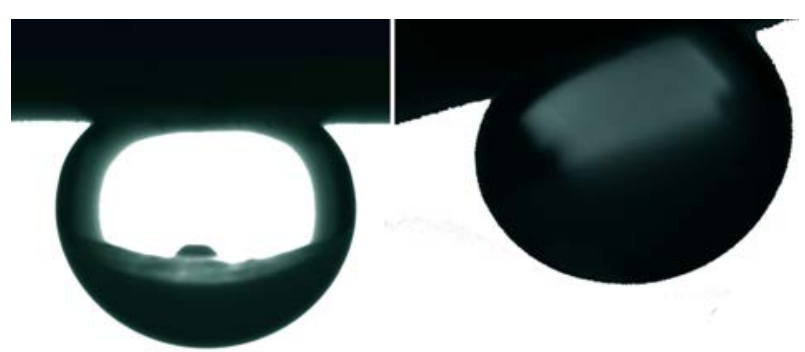

Fig. 1: Pendant mode droplet on a chemically textured substrate (a) horizontal position and, (b) substrate with a $15^{\circ}$ inclination with respect to the horizontal plane. 


\section{Experimental details}

Schematic diagram of the experimental setup is shown in Fig. 2. The setup consists of high speed camera, micro-injection pump, diffused white light source, chemically textured substrate and a substrate holder, with suitable arrangement for its inclination. A high speed camera (Photron-Fastcam ${ }^{\circledR}$ SA-3) and diffused light sources are attached with the substrate holder to ensure that the camera, light and substrate are properly aligned. One end of the substrate holder is hinged and other end can move up/down to locate the substrate in the desired inclination. A single pendant droplet of a given size is first allowed to be formed on the surface oriented at a given inclination from the horizontal. After the first drop is stabilized, a second drop is allowed to slowly grow adjacent to it, at an extremely slow rate by a microsyringe pumping. The micro-syringe pump can deliver a calculated amount of liquid to the drops which grow quasi-statically underneath the substrate, until coalescence process commences with the neighboring static droplet. Apart from high speed videography, laser confocal microscope is independently undertaken to capture the evolution of the foot print of the two drops which are coalescing. The hydrophobic substrate is prepared by developing a monolayer of n-octadecanethiol on it by chemical action. The detail of substrate predation is given below.

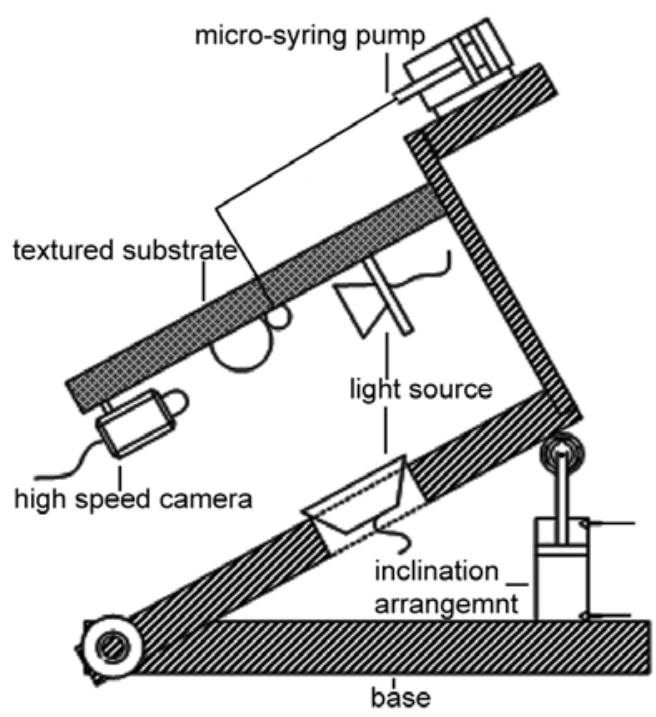

Fig. 2: Schematic diagram of setup used for visualizing coalescence of pendant drop.

\subsection{Substrate preparation}

The substrate is first cleaned with neutral liquid detergent (Labolene ${ }^{\circledR}$-Fischer Scientific), and flushed with acetone and deionized water. The clean copper substrate is then immersed into the etching reagent consisting of 0.85 $\mathrm{mol} / \mathrm{liter} \mathrm{K}_{2} \mathrm{SO}_{4}$ and $3.5 \mathrm{~mol} / \mathrm{liter}$ aqueous solution. The reagent with the copper substrate is kept in furnace at temperature of $70^{\circ} \mathrm{C}$, which results in the following chemical reaction:

$\mathrm{Cu}+2 \mathrm{KOH}+\mathrm{K}_{2} \mathrm{SO}_{4} \rightarrow \mathrm{Cu}(\mathrm{OH})_{2}+2 \mathrm{~K}_{2} \mathrm{SO}_{4}$

When $\mathrm{Cu}(\mathrm{OH})_{2}$ is well formed on the substrate, which take about 30 minutes, the substrate is placed into furnace at temperature of $180^{\circ} \mathrm{C}$ for 2.5 hours, in which it is dehydrated and layer of $\mathrm{CuO}$ is formed.

$\mathrm{Cu}(\mathrm{OH})_{2} \rightarrow \mathrm{CuO}+\mathrm{H}_{2} \mathrm{O}$

The cooper substrate is then immersed into the solution of n-octadecanethiol in ethanol for two hours at constant temperature of $75^{\circ} \mathrm{C}$. In this solution the chemical reaction is as:

$2 \mathrm{HSC}_{18} \mathrm{H}_{17}+\mathrm{CuO} \rightarrow \mathrm{Cu}+\mathrm{H}_{2} \mathrm{O}+\left(\mathrm{SC}_{18} \mathrm{H}_{37}\right)_{2}$

$2 \mathrm{HSC}_{18} \mathrm{H}_{17}+\mathrm{Cu} \rightarrow \frac{1}{2} \mathrm{H}_{2} \mathrm{O}+\mathrm{CH}_{18} \mathrm{H}_{37} \mathrm{SCu}$

$\mathrm{CH}_{18} \mathrm{H}_{37} \mathrm{SCu}$ monolayer is finally formed over the substrate. After the substrate is treated with the method described above, the contact angle of drop is measured in pendant mode at horizontal and inclined substrate, as was shown in Figure 1.

\section{Simulation details}

The schematic diagram of threedimensional model of two drop coalescence underneath an inclined textured substrate is shown in Figure 3. To the first approximation, the initial drop shape is part of sphere. During the coalescence, the flow field sets up within coalescing drops which alters the pressure distribution and hence the shape of drop.

The governing equation for flow, and interface tracking scheme within the framework of incompressible phases (water and air) is:

$$
\frac{\partial \rho}{\partial t}+\vec{u} . \nabla \rho=0
$$

$\rho \frac{\partial \vec{u}}{\partial t}+\rho(\vec{u} . \nabla) \vec{u}=-\nabla \cdot p+\mu\left(\nabla u+\nabla u^{T}\right)+F_{g}+F_{s t}+F_{\text {ext }}$ 
(a)

(b)
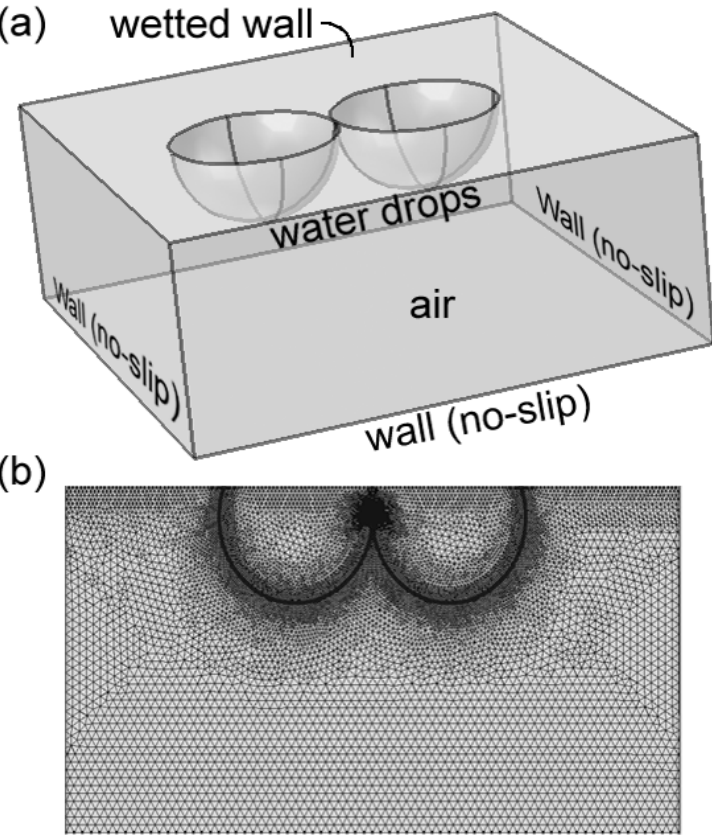

Fig. 3: (a) Schematic diagram of computational domain. Top wall is wetted wall and others are noslip boundary condition. (b) Frontal plane (xy, z = 0) of computational domain with a tetrahedral grid and coordinate system.

The phase field method tracks the interface by adding the flowing equations (Takadaa et al. 2006):

$$
\frac{\partial \phi}{\partial t}+\nabla \cdot(\phi \vec{u})=-\nabla \cdot[\Gamma \phi \nabla \eta]
$$

where $t$ is the time, $\vec{u}$ the flow velocity and $\rho$ is the density of two-phase fluid. In Eq. (2), $g$ is the gravity, $p$ the pressure, $\mu$ the viscosity, and $\rho$ denotes the density in continuous phase. The continuous scalar variable $\phi$ in Eq. (3) is an index to describe the interface profile. In this study, the mobility factor $\Gamma>0$ is set to be constant in the whole flow field for simplicity, and the chemical potential $\eta$ for the inhomogeneous system is derived from a vander-Waals bulk free energy $\psi$ and an excessive energy by $|\nabla \phi|^{2}$ as follows,

$\eta=\frac{\partial \psi}{\partial \phi}-K_{\phi} \nabla^{2} \phi$

where, $K_{\phi}$ is a parameter to control the interfacial thickness.

The surface tension force for the phase field method is implemented as body force $F_{\text {st }}=\eta \nabla \phi$. The gravity force is $F_{g}=V \rho g . F_{e x t}$ and the force arising due to free energy is computed according to, $F_{\text {ext }}=\frac{\partial \psi}{\partial \phi} \nabla \phi$.

The volume of fraction of fluids is computed as:

$V_{f}=\min (\max ([(1+\phi) / 2], 0), 1)$

where, the $<\min >$ and $<\max >$ operators are used so that the volume fraction has a lower limit of 0 and upper limit of 1 . The density and dynamic viscosity are then computed by

$\rho=\rho_{1}+\left(\rho_{2}-\rho_{1}\right) V_{f}$
$\mu=\mu_{1}+\left(\mu_{2}-\mu_{1}\right) V_{f}$

where $\rho_{1}$ and $\rho_{2}$ are densities and $\mu_{1}$ and $\mu_{2}$ are the dynamic viscosities of fluid 1 and fluid 2 respectively. The above equations (1-6) are solved by $\mathrm{COMSOL}^{\circledR}$, finite element based commercial solver.

\section{Results and Discussion}

Drop shapes of water at various times instants during the coalescence event are experimentally obtained as well numerically simulated underneath an inclined textured substrate, as shown in Figure 4. Figure 4(a-b) shows the comparison of foot print of drop captured by simulation and observed by the confocal microscope. Figure 4 (c) shows the drop coalescence process, as observed by high speed camera, at substrate inclination $=45^{\circ}$.
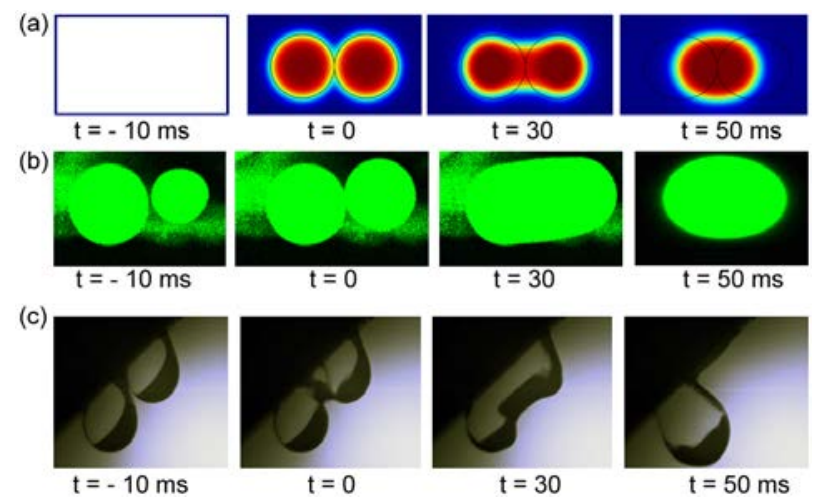

Fig. 4: (a) Foot-print of two coalescing droplets captured in simulation (Comsol ${ }^{\circledR}$ ). (b) Foot-print of two coalescing droplets, as observed by florescence confocal microscopy (c) frontal plane observed by high speed camera, plate inclination $=45^{\circ}$, coalescences of two large drops resulting in criticality and eventual slide-off of the droplet from the inclined surface (both drops $r=2.05 \mathrm{~mm}$ ). 

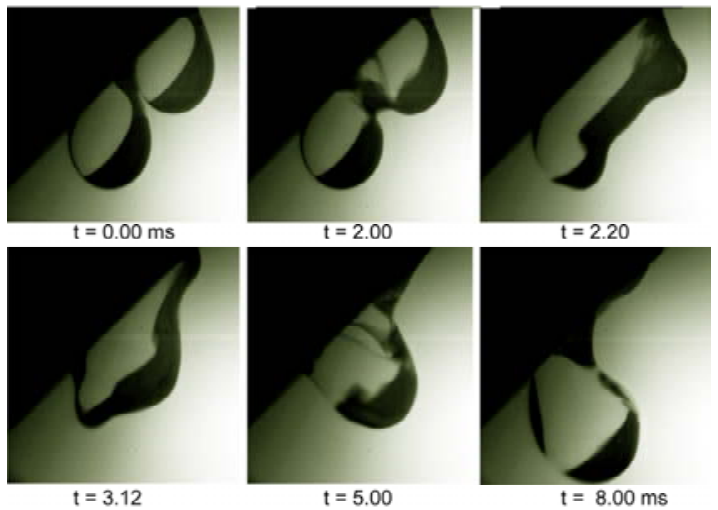

Fig. 5: Coalescence of two pendant water drops of approximately same radius $(r=2.15 \mathrm{~mm}$ and 2.00 $\mathrm{mm}$ respectively) underneath $45^{\circ}$ inclined chemically textured copper substrate.
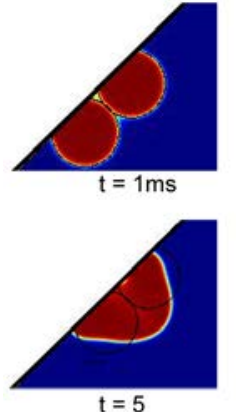
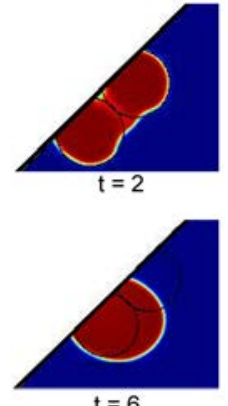

$\mathrm{t}=6$
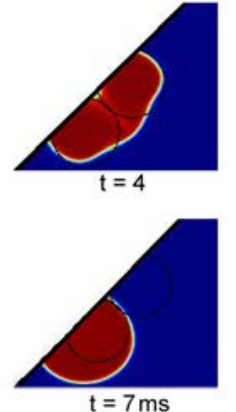

Fig. 6: Simulated image sequence showing coalescence of two pendant droplets of water of approximately same radius (respective $r=2.15 \mathrm{~mm}$ and $2.00 \mathrm{~mm}$ ) underneath $45^{\circ}$ inclined chemically textured copper substrate.

Figure (5-8) show the side view of drop coalescence event during the experiment (high speed camera) and simulation (Comsol®). While qualitative features of drop coalescence are being captured by the simulation, several specific features do not conform to experimental evidence. One of the major issues which generate discrepancy between simulation and experiment is droplet pinning behavior at localized points on the three-phase contact line. Such pinning action has not been incorporated in the simulation model. However, several other features such as liquid bridge formation and qualitative evolution of the droplet shape can indeed be captured by the simulation, as shown in Figures 4-8. It is observed that a liquid bridge is formed at early stage of coalescence. The negative curvature at the liquid bridge drives the coalescence process. The bridge radius grows linearly with time at initial stage of coalescence, but later stage of coalescence grows at a rate proportional to $\left(t^{a}\right)$.

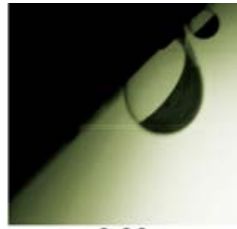

$\mathrm{t}=0.00 \mathrm{~ms}$

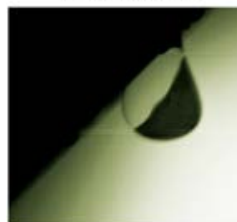

$\mathrm{t}=3.12$
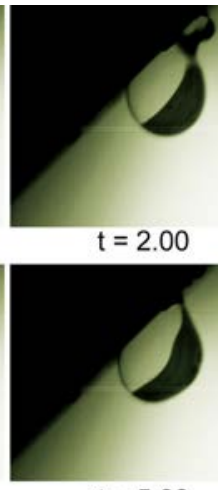

$\mathrm{t}=5.00$

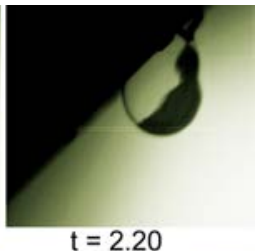

$t=2.20$

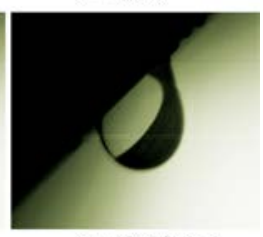

Fig. 7: Coalescence of two pendant droplets of water of different volume (respective $r=2.00 \mathrm{~mm}$ and $0.40 \mathrm{~mm}$ ) underneath $45^{\circ}$ inclined chemically textured copper substrate.

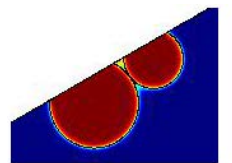

$\mathrm{t}=1 \mathrm{~ms}$

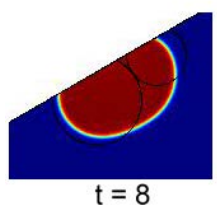

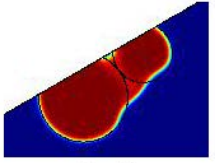

$\mathrm{t}=3$

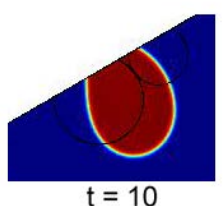

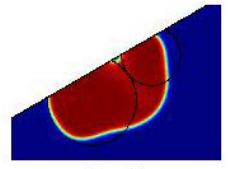

$t=5$

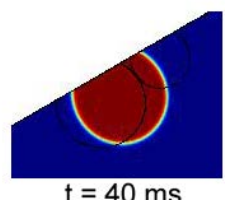

Fig. 8: Simulated image sequence showing coalescence of two pendant droplets of water of different volume $(r=2.00 \mathrm{~mm}$ and $0.40 \mathrm{~mm}$, respectively) underneath $45^{\circ}$ inclined chemically textured copper substrate.

The value of the exponent ' $a$ ' is always found to be less than 1 and depends and on the size and shape of coalescing drops. In the experiment as well as in the simulation, it is found that the footprint of the two drops while they are coalescing takes the shape of a peanut (elongated elliptical shape), as shown in Figure 4(a-b).

The coalescence dynamics thrives on the extra surface energy which gets released in the process. Immediately after coalescence has commenced, the process is limited by viscous and inertia forces. The free surface oscillations of the resulting droplet can last $\sim 10$ to 40 milliseconds, depending on the size of the original droplets and their thermophysical properties. The viscous dissipation close to the three phase contact line will also determine the overall dynamical time scales. Thus, the coalescence process underneath substrates consists of two stages: an initial rapid growth 
of a meniscus bridge between the droplets, and a slow relaxation of the combined droplet shape from ellipsoidal to a more spherical shape at longer times. Simultaneously, the base of the droplet also changes shape as the final profile moves towards equilibrium. It is observed that when two small drops coalesce and the final volume of resulting composite drop is less than the critical size needed for sliding-off, the center of the final drop is the approximately at the weighted average of mass center of the original two individual drops. The triple-phase contact line and the leading and trailing side contact angles of the coalesced drop show periodic damped oscillatory behavior, which is also a function of the substrate inclination. This behavior requires further detailed studies.

Unlike sessile mode, coalescence of two large drops in pendant mode may lead to a critical condition which triggers a slide-off and/or fall-off of the drop from the substrate, as shown in Figure 5. (slide-off leads to eventual fall-off) and Figure 7 (only slide-off). In the early stage of the coalescence underneath the inclined substrate, the initial force balance is broken by the formation of the liquid bridge. The highly concave interfaces at the liquid bridge, as well as the highly convex interface at the retracted top side part of two drops, build the capillary pressure gradient to drive the interior liquid flow. In the later stage of the coalescence, the surface tension gradient resulting from the highly convex interface at the forepart, to the near flattened interface at the back part of the coalesced drop, in conjunction with adverse gravity, induces the interior liquid flow and the oscillation process. Finally, the downhill displacement of the backend of the triple-phase contact line or the entire coalesced drop can occur due to the body force of gravity. Hence, the coalescence process passively promotes accelerated motion of the droplets. On one hand, the retention force arising due to the contact angle hysteresis and viscous dissipation tries to make the drop stable while, on the other hand, the gravity force and energy release due the coalescence tends to induce instability. The resulting equilibrium relaxation time due to the interplay of these forces can reach a few milliseconds, depending on the drop sizes, initial conditions and surface properties (contact angle, roughness and pinning).

Figure (5-6) show the comparison of drop shape captured in simulation and observed by high speed camera during the experiment approximately same size (2.15 $\mathrm{mm}$ and 2.00) of drop of coalescence. If the size of the resulting drop is such that the body forces exceed the retention force then the droplet will start sliding down the substrate. As soon as the drops coalesce, the interface oscillations resulting from the excess interfacial energy available can destabilize the entire droplet and the droplet can fall off from the substrate. As the gravity acts downwards, there is a considerable change in the potential energy of the resulting droplet. This is in contrast to coalescence of sessile droplets.

In Figure (7-8), coalescence of a very small drop with the comparatively larger drop has been experimentally shown and compared with the simulations. In this case, the smaller drops gets sucked inside the larger drop, the resulting drop is still stable (no fall-off). However, the rear contact line is seen to move downwards during the relaxation period. In this way, the final equilibrium is achieved. Another result which needs further exploration is the fact that, in contrast to sessile drop coalescence, in pendant mode coalescence on inclined surfaces, the center of gravity of the resulting equilibrium droplet may not be the weighted average of the original two droplets.

\section{Summary and conclusions}

On the basis of the experiments and simulation of liquid (water) drop coalescence underneath an inclined chemically textured hydrophobic substrate, the following preliminary conclusions can be drawn:

- Drop coalescence commences when two droplets approach each other at the threephase contact lines. Experiments and simulations have been performed with negligible approach velocity of drops.

- A tiny liquid bridge is immediately formed at the commencement of coalescence, induced 
by the van-der-Walls forces, which grows with time as the excess surface energy gets released in the process. Immediately afterwards, the process is limited by viscous and inertia forces, as the drop eventually attains equilibrium. Then extra surface energy gets eventually dissipated through viscous dissipation. In the process, free surface oscillations are clearly seen.

- The typical relaxation time scales of the composite droplet after coalescence is of the order of $\sim 10$ to $100 \mathrm{~ms}$, depending on the size of original two droplets, thermophysical properties of the fluid and the liquid-substrate combination. Experimental time scales are smaller than the corresponding simulation values as the latter does not take the strong local dissipation near the three-phase contacts.

- During the experiments, local pinning action of the three-phase contact line is observed during coalescence and while the droplet relaxes to equilibrium. At present, this localized phenomenon has not been taken into consideration during the simulations. In conjunction with very high dissipation at the three-phase contact line, local pinning is also the reason for higher critical diameters obtained during experiments as compared to the corresponding simulation results.

- The center of mass of the resultant coalesced drop was not equal to the weighted average center of mass of the individual drops, the discrepancy being attributed to external forces, such as those that pin the contact line.

- Immediately after coalesce, the footprint of the droplet is elliptical which eventually relaxes to a circular shape.

- On inclined surfaces, coalescence of drops can lead to a slide-off or fall-off instability. During slide off, the droplet may leave behind a layer of liquid due to local attachment forces.

\section{Acknowledgements}

Financial support from the Board of Research for Nuclear Sciences (BRNS), Mumbai, India to carry out this research work is gratefully acknowledged.

\section{References}

Andrie C., Beysens D.A., Nikolayev V.S., and Pomeau Y., 2002. Coalesnce of sessile drops, J. of Fluid Mech 453, 427-438.

Blanchette F., Messio L., and Bush J. W. M., 2009. The influence of surface tension gradients on drop coalescence, Phys. of Fluids 21, 072107- 072109.

Bordoloi A. D., and Longmire E. K., 2012. Effect of neighboring perturbations on drop coalescence at an interface, Physics of Fluids 24, 062106-062128.

Leach R. N., Stevens F., Langford S. C., and Dickinson J. T., 2006. Dropwise condensation: experiement and simulation of nucleation and growth of water drops in a cooling system, Langmuir 22, 8864-8872.

Liao Q., Zhu X., Xing S.M., and Wang H., 2008. Visualization study on coalescence between pair of water drops on inclined surfaces, Experimental Thermal and Fluid Science 32, 1647-1654.

Eggers J., 1998. Coalescence of spheres by surface diffusion, Physical Review Letters 80, 2634-2637.

Narhe R., Beysens D., and Nikolayev V. S., 2004. Contact line dynamics in drop coalescence and spreading, Langmuir 20, 1213-1221.

Narhe, R., Beysens D., and Nikolayev, V. S., 2005. Dynamics of drop coalescence on a surface: The role of initial conditions and surface properties, Int. J. of Thermophysics 26, 8593-8597.

Rose J. W., 2002. Dropwise condensation Theory and Experiments: A review, Proc. Institution of Mechanical Engineers 216, 115-118.

Sellier M., Nock V., and Verdier C., 2011. Selfpropelling coalescing droplets, Int. J. of Multiphase Flow 37, 462-468.

Sikarwar B. S., Khandekar S., Agrawal S., Kumar S. and Muralidhar K., 2012. Dropwise Condensation Studies on Multiple Scales, Heat Transfer Engineering, 33(4-5), 301-341,

Sikarwar B. S., Battoo N. K., Khandekar S. and Muralidhar K., 2010. Dropwise Condensation underneath Chemically Textured Surfaces: Simulation and Experiments, ASME Journal of Heat Transfer 133(2) 021501 (1-15)

Takadaa N., Misawaa M., and Tomiyamab A., 2006. A phase-field method for interface-tracking simulation of two-phase flows, Mathematics and Computers in Simulation 72, 220-226.

Wang H., Liao Q., Zhu X., Li J. X., and Tian X., 2010, Experimental studies of liquid droplet coalescence on the gradient surface, J. Supercond. and Novel Magnetism 23(6), 1165-1168. 